\title{
From online resources to collaborative global neuroscience research: where are we heading?
}

Pike See Cheah ${ }^{1,2}$, King-Hwa Ling ${ }^{2,3}$ and Eric Tatt Wei Ho ${ }^{4, *}$

\footnotetext{
${ }^{1}$ Department of Human Anatomy, Faculty of Medicine and Health Sciences, Universiti Putra Malaysia.

${ }^{2}$ NeuroBiology \& Genetics Group, Genetics and Regenerative Medicine Research Centre, Faculty of Medicine and Health Sciences, Universiti Putra Malaysia.

${ }^{3}$ Department of Biomedical Sciences, Faculty of Medicine and Health Sciences, Universiti Putra Malaysia.

${ }^{4}$ Center for Intelligent Signal and Imaging Research, Universiti Teknologi PETRONAS, Perak, Malaysia.

*Corresponding authors: hotattwei@utp.edu.my; Tel.: +60-5-368-7899
}

Published: 21 July 2020

https://doi.org/10.31117/neuroscirn.v3i3.51

Keywords: neuroinformatics; machine learning; bioinformatics; brain project; online resources; databases

C2020 by Cheah et al. for use and distribution in accord with the Creative Commons Attribution (CC BY-NC 4.0) license (https://creativecommons.org/licenses/by-nc/4.0/), which permits unrestricted non-commercial use, distribution, and reproduction in any medium, provided the original author and source are credited.

\subsection{INTRODUCTION}

Neuroscience has emerged as a richly transdisciplinary field, poised to leverage potential synergies with information technology. To investigate the complex nervous system in its normal function and the disease state, researchers in the field are increasingly reliant on generating, sharing and analyzing diverse data from multiple experimental paradigms at multiple spatial and temporal scales (Frackowiak \& Markram, 2015). There is growing recognition that brain function must be investigated from a systems perspective. This requires an integrated analysis of genomic, proteomic, anatomical, functional, topological and behavioural information to arrive at accurate scientific conclusions. The integrative neuroinformatics approaches for exploring complex structure-function relationships in the nervous system have been extensively reviewed (Kötter, 2001; Rao et al., 2015). To support neuroscience research, the neuroscientific community also generates and maintains web-accessible databases of experimental and computational data and innovative software tools. Neuroinformatics is an emerging sub-field of neuroscience which focuses on addressing the unique technological and computational challenges to integrate and analyze the increasingly high-volume, multi-dimensional, and finegrain data generated from neuroscience experiments.
The most visible contributions from neuroinformatics include the myriad reference atlases of brain anatomy (human and other mammals such as rodents, primates and pig), gene and protein sequences and the bioinformatics software tools for alignment, matching and identification. Other neuroinformatics initiatives include the various open-source preprocessing and processing software and workflows for data analysis as well as the specifications for data format and software interoperability that allow seamless exchange of data between labs, software tools and modalities.

The tremendous heterogeneity of neuroscience data, reflecting multimodal and multi-scale investigations necessarily dictated by brain complexity and interspecies diversity, delayed the emergence of an information management culture and data sharing policies (Campbell, 2009; Wertheim \& Sidman, 1991). Among the significant challenges were developing consensus on standardization of terminology, configurations for data acquisition instruments and data storage formats which are prerequisites for seamless sharing of information between investigators. Standard terminology is crucial to make information FAIR - findable, accessible, interoperable and reusable (Wilkinson et al., 2016) through metadata annotation which includes entries on other animal species, 
resource type (software, atlas, database), scale (from molecules to the whole brain), application (visualization, analysis, simulation), and measurement type (anatomical, functional, biochemical, and more).

Various datasets have been made freely available to neuroscientists (see Table 1 \& Figure 1 ). In recent years, a growing number of research projects have made freely available functional and anatomical images and recordings of the brain from acquisition modalities such as positron emission tomography (PET), electroencephalography (EEG) and magnetic resonance imaging (MRI). These data acquired in individual labs and studies are increasingly analyzed in conjunction with data from past studies using metaanalytic tools. Polavaram and Ascoli have characterized a representative sample of 337 neuroinformatics resources in terms of several dimensions (Polavaram \& Ascoli, 2015). The collection of openly shared neuroscience datasets and neuroinformatics tools continues to grow rapidly.

In this editorial commentary, we believe it is time to build greater awareness of the co-evolution of neuroscience experimentation with informatics. We highlight representative major data repositories made available as free virtual resources to serve as a starting point for researchers seeking to enrich the scope of their analysis. Subsequently, we summarize the trends that are anticipated to shape the landscape of neuroscience research in the era of open science.

Table 1: List of resources

\begin{tabular}{|c|c|c|c|}
\hline Resources & Species* & Type $^{\#}$ & URL \\
\hline OpenNeuro & $\mathrm{H}$ & $\mathrm{BE}$ & https://openneuro.org/ \\
\hline Human Brain Atlas & $\mathrm{H}$ & A, GE & http://human.brain-map.org \\
\hline $\begin{array}{l}\text { BrainSpan Atlas of the } \\
\text { Developing }\end{array}$ & $\mathrm{H}$ & A, GE & http://www.brainspan.org \\
\hline Aging, Dementia and TBI & $\mathrm{H}$ & $A, B A, G E, P E$ & http://aging.brain-map.org/ \\
\hline The Whole Brain Atlas & $\mathrm{H}$ & $\mathrm{A}, \mathrm{BE}$ & http://www.med.harvard.edu/aanlib/ \\
\hline HUPO Brain Proteome Project & $\mathrm{H}$ & $\mathrm{PE}$ & http://www.hbpp.org \\
\hline The BigBrain & $\mathrm{H}$ & A & http://mcin.ca/research/bigbrain \\
\hline Human Connectome Project & $\mathrm{H}$ & CNT & http://www.humanconnectomeproject.org/ \\
\hline Human Transcriptomic Cell Types & $\mathrm{H}$ & GE & $\begin{array}{l}\text { http://celltypes.brain- } \\
\text { map.org/rnaseq/human/cortex }\end{array}$ \\
\hline IVY Glioblastoma Atlas Project & $\mathrm{H}$ & A, GE & http://glioblastoma.alleninstitute.org \\
\hline The Brain Development & $\mathrm{H}$ & $\mathrm{A}, \mathrm{BE}$ & http://brain-development.org/ \\
\hline $\begin{array}{l}\text { Alzheimer's Disease } \\
\text { Neuroimaging Initiative }\end{array}$ & $\mathrm{H}$ & $\mathrm{BE}$ & http://adni.Ioni.usc.edu/adni-3/ \\
\hline JuBrain Atlas & $\mathrm{H}$ & A & https://jubrain.fz-juelich.de/ \\
\hline GLIMPS & $\mathrm{H}$ & $\mathrm{BE}$ & http://glimpsproject.com/ \\
\hline Adult \& Developing NHP Atlas & MK & $\mathrm{A}, \mathrm{GE}$ & http://www.blueprintnhpatlas.org/ \\
\hline INIA19 Primate Brain Atlas & MK & $A, B E$ & https://www.nitrc.org/projects/inia19/ \\
\hline Brain/MINDS & MK & $\mathrm{A}, \mathrm{BE}$ & https://brainminds.jp/en/ \\
\hline $\begin{array}{l}\text { Marmoset Brain Connectivity } \\
\text { Portal }\end{array}$ & MK & CNT & $\begin{array}{l}\text { http://www.braincircuits.org/marmoset- } \\
\text { home/ }\end{array}$ \\
\hline UNC Primate Brain Atlas & MK & A & https://www.nitrc.org/projects/primate atlas \\
\hline NeuroMap Macaque Atlas & MK & A, CNT & $\begin{array}{l}\text { https://scalablebrainatlas.incf.org/macaque/D } \\
\text { B09 }\end{array}$ \\
\hline 3D Digital D99 Template & MK & A & $\begin{array}{l}\text { https://afni.nimh.nih.gov/pub/dist/atlases/ma } \\
\text { caque/ }\end{array}$ \\
\hline MNI Monkey Brain & MK & A & http://www.bic.mni.mcgill.ca/ServicesAtlases \\
\hline Yerkes19 Macaque Atlas & MK & A & https://balsa.wustl.edu/kNzN2 \\
\hline
\end{tabular}




\begin{tabular}{|c|c|c|c|}
\hline CIVM MRI/DTI Atlas & MK & A & $\begin{array}{l}\text { https://scalablebrainatlas.incf.org/macaque/C } \\
\text { BCetal15 }\end{array}$ \\
\hline NMT Macaque Atlas & MK & A & $\begin{array}{l}\text { https://afni.nimh.nih.gov/pub/dist/atlases/ma } \\
\text { caque/nmt }\end{array}$ \\
\hline Developing Mouse Brain Atlas & $\mathrm{M}$ & A, GE & http://developingmouse.brain-map.org/ \\
\hline Mouse Brain Atlas & M & A, GE & https://mouse.brain-map.org/ \\
\hline Mouse Spinal Cord Atlas & $\mathrm{M}$ & A, GE & http://mousespinal.brain-map.org/ \\
\hline Brain Observatory & M & A, BE & http://observatory.brain-map.org/visualcoding \\
\hline Mouse Brain Connectivity Atlas & $\mathrm{M}$ & A, CNT & http://connectivity.brain-map.org/ \\
\hline Mouse Transcriptomic Cell Types & $\mathrm{M}$ & GE & $\begin{array}{l}\text { http://celltypes.brain- } \\
\text { map.org/rnaseq/mouse/cortex-and- } \\
\text { hippocampus }\end{array}$ \\
\hline Visual Coding with Neuropixels & M & $\mathrm{BE}$ & $\begin{array}{l}\text { https://portal.brain- } \\
\text { map.org/explore/circuits/visual-coding- } \\
\text { neuropixels }\end{array}$ \\
\hline The Mouse Brain Library & $\mathrm{M}$ & A, GE & http://www.mbl.org/ \\
\hline Yongsoo Kim Lab Brain-Atlas & $M$ & A & https://kimlab.io/brain-map/atlas/ \\
\hline Blue Brain Cell Atlas & $\mathrm{M}$ & A, BA & https://bbp.epfl.ch/nexus/cell-atlas/ \\
\hline Mouse Connectome Project & $\mathrm{M}$ & CNT & https://www.nitrc.org/projects/mcp/ \\
\hline Atlas of the Aging Mouse Brain & M & GE & $\begin{array}{l}\text { https://singlecell.broadinstitute.org/single cell } \\
\text { Lstudy/SCP263 }\end{array}$ \\
\hline Tabulo Muris Senis & M & BA & $\begin{array}{l}\text { https://tabula-muris- } \\
\text { senis.ds.czbiohub.org/brain-non-myeloid/facs/ }\end{array}$ \\
\hline
\end{tabular}

\begin{tabular}{|c|c|c|c|}
\hline Hippocampome Portal & $\mathrm{M}$ & $\mathrm{BA}, \mathrm{BE}, \mathrm{PE}$ & http://hippocampome.org/php/index.php \\
\hline Human Protein Atlas & $\mathrm{H}, \mathrm{M}, \mathrm{P}$ & $A, G E, P E$ & $\begin{array}{l}\text { https://www.proteinatlas.org/humanproteom } \\
\text { e/brain }\end{array}$ \\
\hline Cell Types Database & $\mathrm{H}, \mathrm{M}$ & $\mathrm{BA}, \mathrm{BE}, \mathrm{GE}$ & http://celltypes.brain-map.org/ \\
\hline Allen Brain Reference Atlases & $\mathrm{H}, \mathrm{M}$ & A & http://atlas.brain-map.org/ \\
\hline Synaptic Physiology & $\mathrm{H}, \mathrm{M}$ & BA & $\begin{array}{l}\text { https://portal.brain- } \\
\text { map.org/explore/connectivity/synaptic- } \\
\text { physiology }\end{array}$ \\
\hline BIRN & $\mathrm{H}, \mathrm{M}$ & $A, B E$ & https://www.nitrc.org/projects/birn/ \\
\hline Human Brain Project & $\mathrm{H}, \mathrm{MK}, \mathrm{M}$ & $A, B A, B E, G E$ & https://www.humanbrainproject.eu/ \\
\hline Neuromorphos.org & $\mathrm{H}, \mathrm{MK}, \mathrm{M}$ & $\mathrm{A}, \mathrm{BA}$ & http://neuromorpho.org/ \\
\hline BrainMaps & $\mathrm{H}, \mathrm{MK}, \mathrm{M}$ & A & http://brainmaps.org/ \\
\hline
\end{tabular}

*H: human; M: mouse, Mcq: monkey; P: pig

\# A: atlas; BE: brain metabolic imaging, activity or electrophysiology (PET, CT, MRI, EEG; MEG etc.); GE: gene expression (RNAISH, microarray, transcriptome etc.); PE: protein expression (ICC, IHC, mass spectrometry, proteome etc.); BA: molecular, cellular or biochemical analysis; CNT: connectome

\subsection{HUMAN BRAIN RESOURCES}

The Human Brain Project was initiated in the early 1990s by several National Institutes of Health and other United States Government agencies (Huerta et al., 1993), and it indicates a vision complementary to the Human Genome Project. This database embraces all species and recognizes the fundamental principle underlying modern biomedical research, that basic research on all species is needed to contribute to an understanding of the human race and their health (Shepherd et al., 1998). This project supports informatics tools for data at all levels of brain organization, spanning from sequence data (DNA and protein) at the most basic level and brain images at the highest level. It contains databases for microcircuits, fine structures of synapses, neuronal membrane properties, neuronal morphology, and physiological data. 


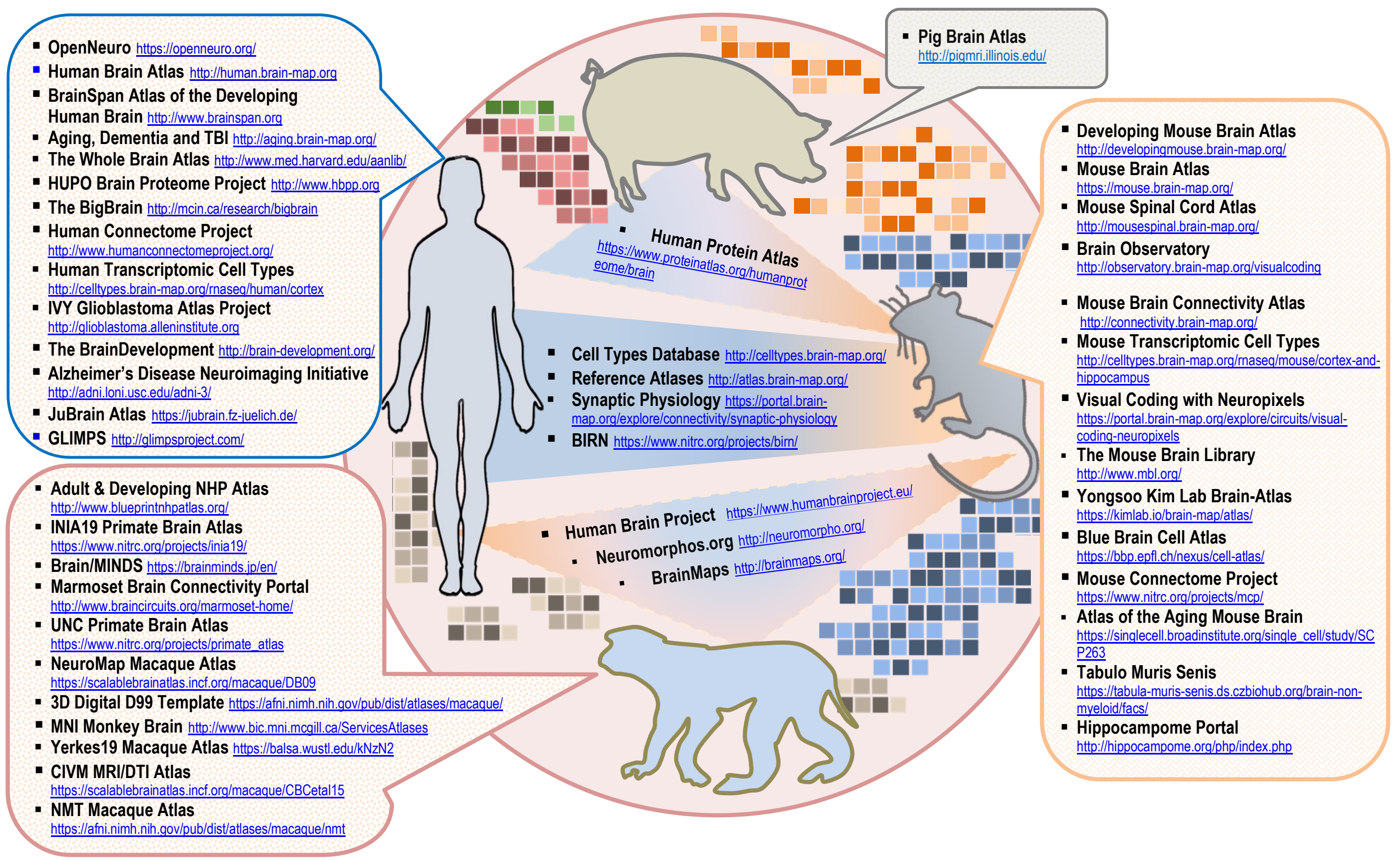

Figure 1: A selected list of publicly available databases encompassing datasets at cellular, tissue, anatomical, functional and disease type levels generated from the human, mouse, pig or/and primate brains. 
The world's first virtual brain, the "Blue Brain" project, was launched and established in 2013, with its simulation performed on the Blue gene supercomputer (built by IBM). The project aims to build biologically detailed digital reconstructions and simulations of the rodents and, ultimately, the human brain (Markram, 2006). The European Union primarily funds the project, which is based on the research strategy developed in the Blue Brain Project. This large-scale 10-year scientific research project aims to reconstruct and simulate the human brain in a supercomputer and so finds cures for psychiatric and neurological disorders. However, the data from the Blue Brain project has yet to be made publicly accessible or open source. The Virtual Brain (known as TVB) is another simulation platform that merges brain structural connectivity on individual brains and then simulates the brain network dynamics. With the healthy brain connectome, TVB facilitates the understanding of particular structurefunction relations in healthy individuals, or study on the effects of plasticity and learning.

Recently, more initiatives were launched all around the world in the race to digitalize the human brain anatomy or function. The BigBrain is an ultrahighresolution three-dimensional (3D) reconstruction of the whole human brain, from cell-body stained histology, with 20 micrometres isotropic resolution. Biomedical Informatics Research Network (BIRN) has developed an infrastructure for the storage of $\mathrm{FMRI}$, MRI scans and atlases for human and mouse brains. The Brain Development is a comprehensive portal that hosts structural MRI images and atlases of fetal, neonatal, paediatric and adult brains, to facilitate computational analysis of brain development.

Combining high-performance computing, simulation and neuroimaging, JuBrain Atlas (Juelich Brain Model) is a research initiative that creates a cytoarchitectonic $3 \mathrm{D}$ and realistic model of the normal human brain. OpenNeuro is a free and openly-available data-sharing platform. It provides a diverse range of human brain imaging data, including the MRI, magnetoencephalography (MEG), EEG, electrocorticography (ECOG), or intracranial electroencephalography (iEEG) data. This open science neuroinformatics database has adopted the straightforward Brain Imaging Data Structure (BIDS) format for shared and curated datasets. This approach facilitates third-party scientists to reanalyze data from published neuroimaging studies and to quantitate differences in the results.
Adding to the list, the Whole Brain Atlas is a collection of representative CNS images including normal and disease atlases [cerebrovascular disease such as stroke or brain attack, degenerative disease, neoplastic disease and inflammatory or infectious disease] that was aimed for educational purposes. The LONI Image and Data Archive (IDA) is a central repository for neuroimaging associated data. It provides the underlying informatics core for The Alzheimer's Disease Neuroimaging Initiative (ADNI), unites researchers to collect, validate and utilize data, aids to define the progression of Alzheimer's disease (AD). The database catalogues MRI and PET images, cognitive tests, genetics, cerebrospinal fluid and blood biomarkers as predictors of AD. Glucose Imaging in Parkinsonian Syndromes is a Dutch multicenter project aims to develop a database containing FDG-PET scans of patients with different neurodegenerative brain diseases including Parkinson's disease, Alzheimer's disease, multiple system atrophy and progressive supranuclear palsy.

\subsection{MOUSE BRAIN RESOURCES}

The Allen Brain Atlas (ABA) project, launched in 2004 by Allen Institute for Brain Science, is one of the highthroughput projects aim to analyze gene expression patterns in the mammalian nervous system systematically. ABA is an online publicly available resource that integrates gene expression and connectivity data with neuroanatomical information for the mouse, human and non-human primate. The $A B A$ involved streamlined high-throughput processes such as animal and tissue processing, riboprobe generation, in situ hybridization (ISH), Nissl staining and anatomic and gene expression quantification. This project strives to gain insight into the temporal and spatial expression of specific genes throughout development and in the adult brain. The value of this dataset for basic neuroscience research, medicine and pharmaceutical drug target development is enormous. $A B A$ has become one of the most crucial reference databases for spatio-temporal analysis of anatomical/histological structures and gene expression.

In 2018, researchers generated a cellular atlas, based on the gene expression profile of 690,000 individual cells, to unmask unique genetic signatures of more than 560 cell populations across nine brain regions of the adult mouse. The Blue Brain Cell Atlas catalogues data from the brain regions account for $\sim 70 \%$ of the total brain volume. This includes telencephalon (frontal and posterior cortex, striatum, hippocampus,), 
diencephalon (thalamus), mesencephalon (substantia nigra, globus pallidus and nucleus basalis, entopeduncular nucleus and subthalamic nucleus) and rhombencephalon (cerebellum). The data is available in an interactive web-based analysis portal called DropViz (Saunders et al., 2018). Instead of months of experiments to determine the gene expression profile of specific cell populations, the data resource facilitates the genetic studies, particularly, the psychiatric and neurological disorders, which usually involved the role of more than one gene.

Recently, Kim's Laboratory adopted the anatomical labels from Franklin and Paxinos into the common coordinate framework (Allen Institute for Brain Science) to merge two labels in a single atlas framework. These highly-segmented anatomical labels, in a common spatial context, help to avoid the inconsistencies in anatomical delineations and nomenclatures, leading to more precise interpretation and integration of neuroscience research (https://kimlab.io/brain-map/atlas/).

The Mouse Connectome Project creates a complete mesoscale 3D digital connectome atlas of the C57Black/6J mouse brain, and to subsequently generate the global neural networks. The Mouse Brain Library consists of high-resolution images and databases of the brain from various genetically characterized strains of mice from both embryonic (gestation day 16 and 18) and adult mouse brain atlases. There are a few portals that catalogue data are focusing on specific brain region instead of the whole mouse brain. Hippocampome Portal is a curated knowledgebase of neuron types in the normal adult rodent hippocampal formation, includes dentate gyrus, cornu ammonis (CA)-1, CA2, CA3, subiculum, and entorhinal cortex. The data available includes cell morphology, electrophysiology, molecular markers, region makeup and connectivity.

Two single-cell transcriptomic atlases of the ageing mouse brain were created to gain better insights into the ageing brain involving both molecular and cellular processes (The Tabula Muris Consortium et al., 2019; Ximerakis et al., 2019). The Atlas of the Aging Mouse Brain catalogues comprehensive datasets of ageingrelated genes, top marker genes, and pathways and ligand-receptor interactions. On the other hand, the Tabula Muris Senis, (The Tabula Muris Consortium et al., 2019) provides a powerful resource of the singlecell transcriptomic atlas across the lifespan of postnatal, adult and ageing mice (6 weeks to 24 months) of 18 tissues and organs (including the brain). The portal provides a wealth of new molecular information related to cell type-specific manifestations of different hallmarks of ageing.

\subsection{WHERE ARE WE HEADING?}

We believe that more neuroscience questions will attempt to be answered through frameworks of analysis on pooled datasets, especially with the trend towards datasets enriched with multimodal data acquisition. Researchers will soon be overwhelmed with diverse and large datasets, and making sense of them would be challenging. For instance, the emergence of various in situ sequencing technologies such as FISSEQ (Lee et al., 2015) and STARmap (Wang et al., 2018) for both 2D and 3D in situ tissue sequencing, respectively, will soon lead to an unprecedented amount of fusion datasets involving 2D/3D brain reconstructions from images with spatial transcriptomic information at single-cell resolution. With the explosion of brain imaging, mapping, clinical, biomarker and connectomics data in recent years, reasonable interpretations of new experimental data should no longer be formulated in isolation from this growing corpus of publicly available and distributed datasets. To meet the high standards of statistical significance required for inferring conclusions on complex and multi-factorial questions in neuroscience studies, there are growing efforts to jointly analyze datasets pooled from multiple studies for example, using meta-analysis techniques. Rather than rendering experiments in small labs obsolete, this trend makes it more crucial for small labs to focus on high quality data-acquisition and to be recognized for contributing findable, accessible, interoperable and reusable (FAIR) data.

To promote these novel use cases for data collaborations in the service of neuroscientific discovery, experimental data must be of high quality with complete annotations on the data and experimental configurations. The data and associated annotations must be stored in formats that promote searchable, retrievable and reusable exchange between computing platforms. Software tools must foster consistent, reproducible preprocessing and analysis results. Open-source software workflows will play an increasingly critical role in neuroscience analysis as the infrastructure that enables both the complex chain of preprocessing required for harmonization of data from across different acquisition scenarios and the objective evaluation of aggregated data against hypotheses. In tandem with the increasing 
complexity and diversity data, hypothesis generation will also evolve towards a greater reliance on computationally-derived scenarios integrated with artificial intelligence models to identify patterns of correlation and causality. Among global neuroscience communities, the International Neuroinformatics Coordinating Facility (INCF) has recently begun undertaking this crucial role to evaluate and endorse standards in data exchange and software workflows as well as best practices in data acquisition, management and analysis (Abrams et al., 2019). However, this endeavour is not to be shouldered by a single organisation alone. For the vision of data-collaborative neuroscience to prosper, it is necessary for the global neuroscience community to actively engage in establishing the landscape of tool and data standards either as a contributor of software tools, datasets or software workflow recipes, as an advocate through the preparation of training resources or as a user participating in the democracy of standardization through constructive feedback and votes.

Neuroscience research has benefited from the integration with informatics approaches which enhanced crosstalk between researchers, data modalities and analysis algorithms. It has also revealed a potential approach to tackle the big science of understanding the brain through greater adoption of open source and open science principles, which began with the open sharing of brain-related datasets online and has now evolved to include software tools, data formats and software workflow recipes. We hope this editorial contribution will play a part in spreading awareness in support of this collaborative vision for global neuroscience research.

Conflicts of Interest: The authors declare no conflict of interest.

\section{References}

Abrams, M., Bjaalie, J., Das, S., Egan, G., Ghosh, S., Goscinski, W., Grethe, J., Kotaleski, J., Ho, E., Kennedy, D., Lanyon, L., Leergaard, T., Mayberg, H., Milanesi, L., Mouček, R., Poline, J.-B., Roy, P., Tang, T., Tiesinga, P., ... Martone, M. (2019). A standards organization for Open and FAIR neuroscience: the International Neuroinformatics Coordinating Facility. OSF Preprints. https://doi.org/10.31219/OSF.IO/3RT9B

Campbell, P. (2009). Data's shameful neglect. Nature, 461(7261), 7261. https://doi.org/10.1038/461145a

Frackowiak, R., \& Markram, H. (2015). The future of human cerebral cartography: A novel approach. Philosophical Transactions of the Royal Society B: Biological Sciences, 370(1668). https://doi.org/10.1098/rstb.2014.0171

Huerta, M. F., Koslow, S. H., \& Leshner, A. I. (1993). The Human Brain Project : an international resource. Trends in Neurosciences, 16(11), 436-438. https://doi.org/10.1016/0166-2236(93)90069-X

Kötter, R. (2001). Neuroscience databases: Tools for exploring brain structure-function relationships. Philosophical Transactions of the Royal Society B: Biological Sciences, 356(1412), 1111-1120. https://doi.org/10.1098/rstb.2001.0902

Lee, J. H., Daugharthy, E. R., Scheiman, J., Kalhor, R., Ferrante, T. C., Terry, R., Turczyk, B. M., Yang, J. L., Lee, H. S., Aach, J., Zhang, K., \& Church, G. M. (2015). Fluorescent in situ sequencing ( FISSEQ ) of RNA for gene expression profiling in intact cells and tissues. Nature Protocols, 10(3), 442-458. https://doi.org/10.1038/nprot.2014.191

Markram, H. (2006). The Blue Brain Project. Nature Reviews Neuroscience, 7, 153-160. https://doi.org/1038/nrn1848

Polavaram, S., \& Ascoli, G. (2015). Neuroinformatics. Scholarpedia, 10(11), 1312. https://doi.org/10.4249/scholarpedia.1312

Rao, R. A., Cecchi, G. A., \& Kaplan, E. (2015). Editorial: Towards an integrated approach to measurement, analysis and modeling of cortical networks. Frontiers in Neural Circuits, 9(OCTOBER), 1-4. https://doi.org/10.3389/fncir.2015.00061

Saunders, A., Macosko, E. Z., Wysoker, A., Brumbaugh, S., Kulp, D., Mccarroll, S. A., Saunders, A., Macosko, E. Z., Wysoker, A., Goldman, M., \& Krienen, F. M. (2018). Molecular Diversity and Specializations among the Cells of the Adult Mouse Brain Resource Molecular Diversity and Specializations among the Cells of the Adult Mouse Brain. Cell, 174(4), 1015-1030. https://doi.org/10.1016/j.cell.2018.07.028

Shepherd, G. M., Mirsky, J. S., Healy, M. D., Singer, M. S., Skoufos, E., Hines, M. S., Nadkarni, P. M., \& Miller, P. L. (1998). The Human Brain Project : neuroinformatics tools for integrating, searching and modeling multidisciplinary neuroscience data. Trends in Neurosciences, 21(11), 460-468. https://doi.org/10.1016/s0166-2236(98)01300-9

The Tabula Muris Consortium, Pisco, A. O., \& Schaum, N. (2019). A Single Cell Transcriptomic Atlas Characterizes Aging Tissues in the Mouse. BioRxiv. https://doi.org/https://doi.org/10.1101/661728

Wang, X., Allen, W. E., Wright, M. A., Sylwestrak, E. L., Samusik, N., Vesuna, S., Evans, K., Liu, C., Ramakrishnan, C., Liu, J., Nolan, G. P., Bava, F., \& Deisseroth, K. (2018). Three-dimensional intact-tissue sequencing of single-cell transcriptional states. Science, 361(6400), eaat5691. https://doi.org/10.1126/science.aat5691

Wertheim, S. L., \& Sidman, R. L. (1991). Databases for neuroscience. Nature, 354(6348), 88-89. https://doi.org/10.1038/354088a0

Wilkinson, M. D., Dumontier, M., Aalbersberg, Ij. J., Appleton, G., Axton, M., Baak, A., Blomberg, N., Boiten, J. W., da Silva Santos, L. B., Bourne, P. E., Bouwman, J., Brookes, A. J., Clark, T., Crosas, M., Dillo, I., Dumon, O., Edmunds, S., Evelo, C. 
T., Finkers, R., ... Mons, B. (2016). Comment: The FAIR Guiding Principles for scientific data management and stewardship. Scientific Data, 3(1), 1-9. https://doi.org/10.1038/sdata.2016.18

Ximerakis, M., Lipnick, S. L., Innes, B. T., Simmons, S. K., Adiconis, X., Dionne, D., Mayweather, B. A., Nguyen, L., Niziolek, Z., Ozek, C., Butty, V. L., Isserlin, R., Buchanan, S. M., Levine, S. S., Regev, A., Bader, G. D., Levin, J. Z., \& Rubin, L. L. (2019). Single-cell transcriptomic profiling of the aging mouse brain. Nature Neuroscience, 22(10), 1696-1708.

https://doi.org/10.1038/s41593-019-0491-3 